\title{
STUDY OF A TURBULENT NITROGEN-DILUTED HYDROGEN-AIR DIFFUSION FLAME THROUGH LARGE-EDDY SIMULATIONS COUPLED WITH A FIRST ORDER CONDITIONAL MOMENT CLOSURE METHOD
}

\author{
A. D`Ausilio*, I. Stanković, B. Merci \\ Department of Flow Heat and Combustion Mechanics, Ghent University, Ghent, Belgium \\ *Correspondence author. Email: alessandro.dausilio@ugent.be
}

\begin{abstract}
Large Eddy Simulations (LES) and Conditional Moment Closure (CMC) as turbulent combustion model. In order to explore the effect of turbulence, two types of inlet boundary conditions are applied: White Noise and a method of Random Spots. The analysis of Favre-averaged profiles of velocity, mixture fraction, temperature and species has led to the conclusion that the method of Random Spots is in much better agreement with the experimental data, as expected. However, several discrepancies between simulations and experiments can also be caused by the boundary conditions applied at the sides and the outlet of the domain.
\end{abstract}

\section{INTRODUCTION}

Hydrogen plays an important role as a fuel or fuel component in the development of new high efficiency combustion devices, with the aim of minimizing pollutant emissions. Therefore it is important to develop reliable models for turbulent combustion of hydrogen containing fuels. In the present study, we apply Large Eddy Simulations (LES) techniques with the first order Conditional Moment Closure (CMC) method [Klimenko 1999 - Garmory 2015] to a nitrogen-diluted hydrogen flame (H3 flame) [Meier 1996]. The LES equations are solved using the OpenFOAM code (OpenFOAM® - The Open Source Computational Fluid Dynamics (CFD) Toolbox). CMC equations are solved using an in-house finite-volume code [Garmory 2015, Zhang 2016].

There are several studies, both experimental [Pfuderer 1996] and numerical, concerning this flame. Pitsch [1998] numerically examined the H3 flame to validate the unsteady flamelet approach coupled with a standard k- $\varepsilon$ model. Results showed that the maximum temperature along the centerline is shifted downstream compared to the experimental results. This discrepancy was attributed to the under-prediction of the spreading rate, related to the turbulence field description. Moreover the temperature was over-predicted in a region far from the inlet.

Using the same flamelet libraries, Forkel [2000] carried out LES of the $\mathrm{H} 3$ flame testing two different inlet boundary conditions. The first one was obtained imposing the instantaneous velocity profile of fully developed pipe flow at the exit plane of the nozzle, while the second was obtained adding random fluctuations to the mean velocity inlet profile. Neither of the methods were able to describe 
properly turbulence fluctuations close to the nozzle. In addition, the position of the onset of decay of mean velocity and mixture fraction on the symmetry axis did not match the experimental value.

Panjvani [2010], performing LES coupled with the Eddy Dissipation Concept [Magnussen 2000], identified the inflow boundary condition as the main cause of the discrepancies between experimental and simulation results .

The H3 flame studies reviewed so far, however, do not take into account the differential diffusion effect, which according to Meier [1996] should be taken into account. Hydrogen diffusivity, both as a molecule and as a radical, is significantly higher than for the other species involved into the combustion mechanism. Nevertheless, in the modeling of turbulent gaseous diffusion flames it is often assumed that molecular diffusivities of species are equal, i.e., the Schmidt numbers are equal. Molecular diffusion is not negligible, though, when the mixing layer is thinner than the smallest turbulent eddies. In such conditions, the assumption of equal molecular diffusivities in turbulent reacting flows affects temperature and species predictions, particularly close to the nozzle. This effect was clearly reported by Maragkos [2014].

Despite this observation, the conventional approach of equal diffusivity is adopted here, because this study is a first step, focusing on the impact of boundary conditions (at the inlet, as well as the sides and the outlet of the computational domain). Adding differential diffusion in the modeling is considered beyond the scope of this study (but it will be an interesting next step to consider).

The principal objective of the present study is to investigate the influence of the turbulent inlet boundary conditions on profiles in physical space. Mean flow fields, mixing fields and temperature fields, as well as fluctuations thereof, are discussed. To that purpose, a method of random spots is used [Kornev 2007]. This method is based on the idea that turbulent flow is a motion of turbulent spots of a certain size arising at random positions at random times. These results are compared to LES-CMC simulation results where turbulent white noise is used as inlet boundary condition. Finally, also the effect of the boundary conditions at the sides and the outlet of the domain is discussed.

The paper is divided into four parts. The first section deals with the mathematical modeling of the LES-CMC. The second section describes the experimental and computational details. The third section focuses on the numerical results, and this is followed eventually by the conclusions.

\section{LES-CMC MODELING}

The LES governing equations for mass, momentum and mixture fraction read [Poinsot 2001]:

$\frac{\partial \bar{\rho}}{\partial t}+\frac{\partial\left(\bar{\rho} \tilde{u}_{i}\right)}{\partial x_{i}}=0$

$\frac{\partial \bar{\rho} \tilde{u}_{i}}{\partial t}+\frac{\partial\left(\bar{\rho} \tilde{u}_{i} \tilde{u}_{j}\right)}{\partial x_{j}}=-\frac{\partial \bar{p}}{\partial x_{i}}+\frac{\partial \widetilde{\tau_{i j}}}{\partial x_{j}}-\frac{\partial \tau_{i j}^{s g s}}{\partial x_{j}}+\bar{\rho} g_{i}$

$\frac{\partial(\bar{\rho} \tilde{f})}{\partial t}+\frac{\partial \bar{\rho} \tilde{u}_{j} \tilde{f}}{\partial x_{j}}=\frac{\partial}{\partial x_{j}}\left(\bar{\rho} D \frac{\partial \tilde{f}}{\partial x_{j}}-\bar{\rho} J_{j}{ }^{s g s}\right)$

In the equations $\bar{\rho}$ is the filtered density, $\tilde{u}$ is the filtered velocity and $\tilde{f}$ is the filtered mixture fraction.

The third term on the right hand side of the Eq.(2), $\tau_{i j}^{s g s}=\bar{\rho}\left(\widetilde{u_{i} u_{j}}-\tilde{u}_{i} \tilde{u}_{j}\right)$, is the sub-grid scale, SGS (residual) stress tensor. Through this term the effect of the small scales is included. The sub-grid scale stresses are expressed according to the Boussinesq [1877] assumption as:

$\tau_{i j}^{s g s}=\bar{\rho}\left(\widetilde{u_{i} u_{j}}-\tilde{u}_{i} \widetilde{u_{j}}\right)=\mu_{t}\left[\left(\frac{\partial \widetilde{u_{i}}}{\partial x_{j}}+\frac{\partial \tilde{u}_{j}}{\partial x_{i}}\right)-\frac{2}{3} \frac{\partial \widetilde{u_{k}}}{\partial x_{k}} \delta_{i j}\right]$

The turbulent viscosity, $\mu_{t}$, is then modeled through the dynamic One Equation Model [Schumann 1975]: 
$\mu_{t}=\bar{\rho} c_{k} \Delta \tilde{k}^{\frac{1}{2}}$

In Eq.(5) $c_{k}$ is a model parameter, determined dynamically [Davidson 1997], $\Delta$ is the cubic root of the LES cell volume and $\tilde{k}$ is the sub-grid scale turbulent kinetic energy, for which a transport equation is solved:

$\frac{\partial(\bar{\rho} \widetilde{k})}{\partial t}+\frac{\partial\left(\bar{\rho} \tilde{u}_{i} \tilde{k}\right)}{\partial x_{i}}=\frac{\partial}{\partial x_{i}}\left[\left(\mu+\frac{\mu_{t}}{S c_{t}}\right) \frac{\partial \tilde{k}}{\partial x_{i}}\right]+P-\bar{\rho} \widetilde{\varepsilon}$

where $P$ is the production rate of the sub-grid scale kinetic energy calculated as:

$P=2 \mu_{t}\left[\tilde{S}: \tilde{S}-\frac{1}{3}\left(\frac{\partial u_{i}}{\partial x_{j}}\right)^{2}\right]-\frac{2}{3} \bar{\rho} \tilde{k} \frac{\partial \widetilde{u}_{i}}{\partial x_{j}}$

And dissipation rate, $\widetilde{\varepsilon}$, is expressed as:

$\widetilde{\varepsilon}=c_{\varepsilon} \widetilde{k}^{3 / 2} \Delta^{-1}$

In Eq.(8) $c_{\varepsilon}$ is a model parameter set equal to 1.05 [Fureby 1997].

In Eq.(3), $D=v / S c$ is the diffusivity, where a constant Schmidt number $S c=0.7$ is used, and $J_{j}{ }^{\text {sgs }}$ is the scalar transport due to the sub-grid scale fluctuations, which is modelled as:

$J_{s g s}=-D_{t} \frac{\partial \tilde{f}}{\partial x_{j}}$

where $D_{t}$ is the turbulent diffusivity: $D_{t}=\frac{v_{t}}{S c_{t}}, v_{t}=\frac{\mu_{t}}{\bar{\rho}}$ and $S c_{t}$ is turbulent Schmidt number. A constant value of $S c_{t}=0.7$ is used throughout this work.

For combustion and turbulence - chemistry interaction, the first order CMC approach is adopted [Klimenko 1999] . CMC model assumes that fluctuations of species mass fractions and enthalpy (or temperature) can be associated with the fluctuation of one scalar (mixture fraction) in non-premixed combustion. This means that the transport equations are solved for the conditional averages, with the conditioning being on the mixture fraction.

The $\mathrm{H}_{2} / \mathrm{O}_{2}$ reaction mechanism as presented by $\mathrm{Li}$ [2004] is used in the present study. It consists of 19 reversible elementary reactions between 9 species $\left(\mathrm{H}_{2}, \mathrm{~N}_{2}, \mathrm{O}_{2}, \mathrm{H}_{2} \mathrm{O}, \mathrm{H}, \mathrm{O}, \mathrm{OH}, \mathrm{HO}_{2}, \mathrm{H}_{2} \mathrm{O}_{2}\right)$. Defining the conditionally filtered mass fraction of species as $Q_{\alpha}=\widehat{Y_{\alpha} \mid \eta}, \alpha=1, \ldots n$, and the conditionally filtered enthalpy as $Q_{h} \equiv \widetilde{h \mid \eta}$, conditioned on the sample space variable, $\eta$, for mixture fraction, the three-dimensional CMC equations read:

$$
\begin{aligned}
& \frac{\partial Q_{\alpha}}{\partial t}+\widetilde{u_{i} \mid \eta} \frac{\partial Q_{\alpha}}{\partial x_{i}}=\widetilde{N \mid \eta} \frac{\partial^{2} Q_{\alpha}}{\partial \eta^{2}}+\widetilde{w_{\alpha} \mid \eta}+e_{f} \\
& \frac{\partial Q_{h}}{\partial t}+\widetilde{u_{i} \mid \eta} \frac{\partial Q_{h}}{\partial x_{i}}=\widetilde{N \mid \eta} \frac{\partial^{2} Q_{h}}{\partial \eta^{2}}+e_{f}
\end{aligned}
$$

It should be highlighted that the CMC equation for the conditionally filtered total enthalpy in Eq.(11) differs from Eq.(10) only for the absence of the chemical source term [Zhang 2016].

The individual terms in Eq.(10), starting from the first term on the left hand side, are: unsteady term, convection, micro-mixing, conditionally filtered chemical source term and sub-grid conditional flux. The latter accounts for the conditional transport in physical space and is modelled with the gradient model [Zhang 2014]:

$e_{f}=\nabla \cdot\left(D_{t} \nabla Q_{\alpha}\right)$ 
As conditional fluctuations of the reactive scalars are much smaller than unconditional fluctuations and can be neglected, a first order closure of the chemical source term can be applied, using the conditional averages of the scalars:

$\widetilde{w_{\alpha} \mid \eta}=w_{\alpha}\left(Q_{\alpha}, Q_{h}\right)$

$\widetilde{u_{\imath} \mid \eta}$ and $\widetilde{N \mid \eta}$ correspond, respectively, to be conditionally filtered velocity and conditionally filtered scalar dissipation rate. These terms are unclosed and therefore require modelling. The conditional velocity is assumed equal to the local unconditional filtered velocity, $\widetilde{u_{i} \mid \eta}=\widetilde{u_{i}}$, according to Garmory [2013]. The conditionally filtered scalar dissipation rate is modeled by the Amplitude Mapping Closure (AMC) model [O’Brien 1991]:

$\widetilde{N \mid \eta}=\frac{\widetilde{N} G(\eta)}{\int_{0}^{1} \tilde{P}(\eta) G(\eta) d \eta}$

In Eq. (14), $\tilde{P}(\eta)$ is the Filtered probability Density Function (FDF), assumed to have a $\beta$-function shape, and $G(\eta)$ is prescribed to be an error function:

$G(\eta)=\exp \left(-2\left[\operatorname{erf}^{-1}(2 \eta-1)\right]^{2}\right)$

The filtered scalar dissipation rate $\widetilde{N}$ is calculated through [Pera 2006]:

$\widetilde{N}=\widetilde{N_{\text {res }}}+\widetilde{N_{s g s}}=D \frac{\partial \tilde{f}}{\partial x_{k}} \frac{\partial \tilde{f}}{\partial x_{k}}+\frac{1}{2} C_{N} \frac{\mu_{s g s}}{\Delta^{2}} \widetilde{f^{\prime \prime 2}}$

In Eq. (16), $C_{N}$ is a constant chosen equal to 42 according to Garmory [2011], and the mixture fraction variance $\widetilde{f^{\prime \prime 2}}$ is calculated as:

$\widetilde{f^{\prime \prime 2}}=C_{V} \Delta^{2} \nabla \tilde{f} \cdot \nabla \tilde{f}$

The main advantage of CMC is that conditional variables vary much less in physical space than unconditional quantities do. Therefore, in order to take full advantage of the LES-CMC formulation, it is common practice to solve the CMC equations on a coarser mesh [Stankovic 2013, Garmory 2015]. This approach is followed. It implies that the flow field information from the LES resolution must be transferred to the CMC resolution and an averaging procedure has to be used. As in previous studies [Stankovic 2013], mass weighted averaging over all LES cells associated with one CMC cell is used for mixture fraction, mixture fraction variance and scalar dissipation rate. For the scalar dissipation rate, the AMC model is applied at the CMC resolution in order to obtain the conditional scalar dissipation rate. The filtered velocity and turbulent diffusivity do not undergo an averaging operation: their values at CMC faces are interpolated using the LES face properties.

Using the local FDF, the resolved temperature and density are calculated from the conditional averages in each LES cell at each time step by integrating over mixture fraction space:

$\frac{1}{\bar{\rho}}=\int_{0}^{1} \frac{1}{\overline{\rho \mid \eta}} \tilde{P}(\eta) d \eta, \tilde{T}=\int_{0}^{1} \widetilde{T \mid \eta} \tilde{P}(\eta) d \eta, \widetilde{Y_{\alpha}}=\int_{0}^{1} \widetilde{Y_{\alpha} \mid \eta} \tilde{P}(\eta) d \eta$

The filtered density and temperature are provided to the LES resolution and the flow field is updated accordingly. This bi-directional data communication between the fine LES mesh and the course CMC mesh is illustrated in Figure 1 [Zhang 2016]. 


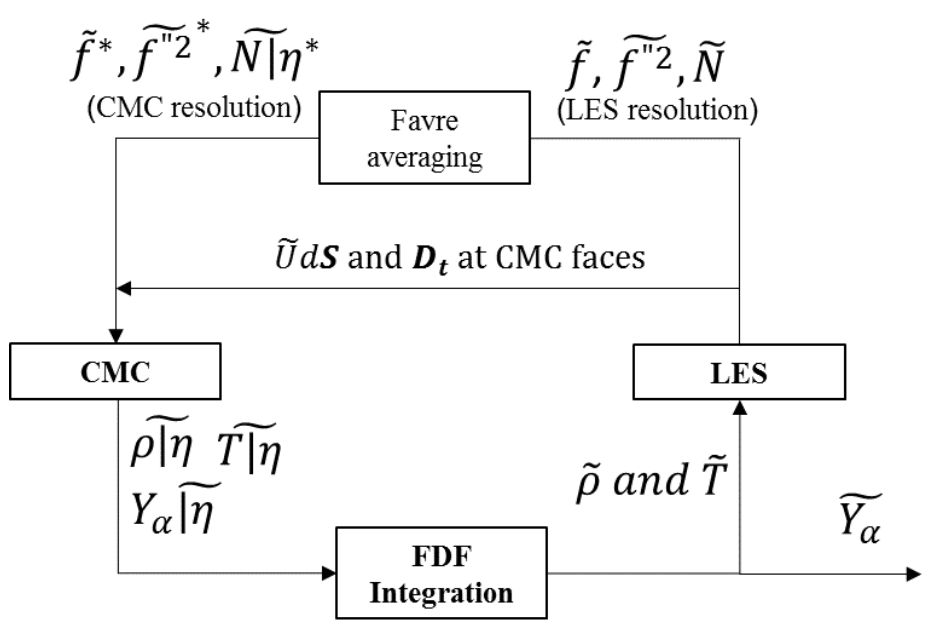

Figure 1. Coupling of LES and CMC [Zhang 2016].

\section{TEST CASE AND NUMERICAL SET-UP}

The non-premixed jet flame is a $H_{2}$ flame, diluted with $50 \%$ vol $N_{2}$ [Meier 1996a]. The experimental data set, available in literature [Meier 1996b], includes simultaneous measurements of velocity, temperature, the major species mass fractions $\left(\mathrm{O}_{2}, \mathrm{H}_{2}, \mathrm{~N}_{2}, \mathrm{H}_{2} \mathrm{O}\right)$, and the mass fraction of $\mathrm{OH}$. In the experiment the burner is a straight tube with inner diameter $d=8 \mathrm{~mm}$ centered in a co-flow emanating from a contoured nozzle. The co-flow air velocity is $0.2 \mathrm{~m} / \mathrm{s}$. Both co-flow air and fuel exit temperature are $298 \mathrm{~K}$.

At the nozzle exit the bulk velocity is $34.8 \mathrm{~m} / \mathrm{s}$ and $\mathrm{Re}=10000$. The mean velocity and the Reynoldsstress components are known only on the axis and as states Forkel [2000] assumption of fully developed turbulent pipe flow could be not correct. The stoichiometric mixture fraction for this fuel composition is $f_{s t}=0.31$ and the adiabatic flame temperature is equal to $\mathrm{T}=2040 \mathrm{~K}$.

The simulation domain consists of a cylinder with radius equal to $11 d$ and length equal to $65 d$, as shown in Figure 2a. The LES mesh has a structured O-ring arrangement. As can be seen in Figure 2c the inlet patch is divided in $4 \times 4$ cells in the rectangular section and $16 \times 16$ in the cylindrical. The coflow patch, reported in Figure 2b, is divided in in 16x50 cells. Axially the domain is discretized using 400 cells. This discretization gives a total number of 0.440 million cells for the whole domain.

a)

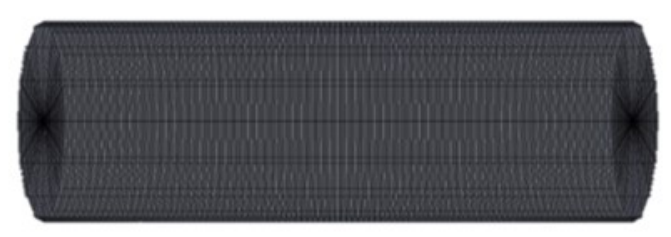

b)

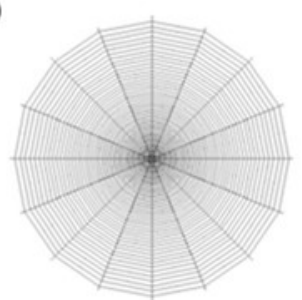

c)

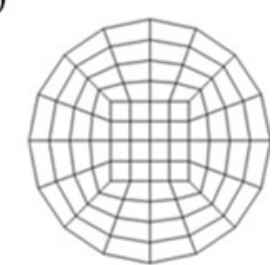

Figure 2. LES mesh used for simulations: (a) whole domain; (b) inlet and co-flow together (c) only inlet.

The CMC grid, reported in Figure 3, consists of $36 \times 40$ cells (radial $\mathrm{x}$ axial), with a total number of 1440 cells. This value has been chosen considering an advantageous trade-off between numerical accuracy and computational cost. In fact, using the same numerical set-up, simulation results obtained with the CMC mesh with 1440 cells were compared with simulation results obtained using a CMC grid with a total number of 3000 cells. No significant differences were noticed comparing the conditional temperature profile at several locations. The mixture fraction sample space is discretized into 100 bins, with a greater concentration of points around the stoichiometric mixture fraction. 
a)

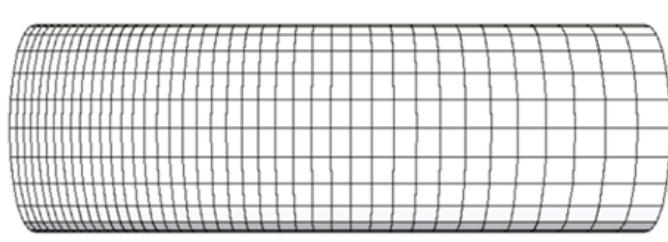

b)

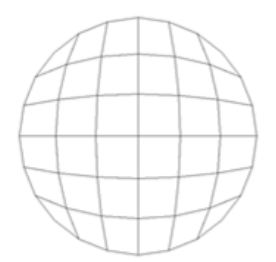

Figure 3. CMC mesh used for simulations (a) whole domain (b) inlet and co-flow together.

As mentioned above, the LES equations are solved using the OpenFOAM ${ }^{\circledR}$ code $\left(\right.$ OpenFOAM ${ }^{\circledR}$ The Open Source Computational Fluid Dynamics (CFD) Toolbox). CMC equations are solved using an in-house finite-volume code [Garmory 2015, Zhang 2016].

The LES equations in reactingFoam solver are discretized using the built-in numerical schemes available in OpenFOAM ${ }^{\circledR}$. They are advanced in time using a first order bounded implicit 'Euler' scheme. The convective terms for the momentum equation are second order centrally differenced using 'Gauss linear' interpolation. The convective term in the mixture fraction equation, a Gauss linear scheme that limits towards upwind in regions of rapidly changing gradient is used. The gradient terms are evaluated through the standard Green-Gauss method. For the diffusion term in the momentum equation the Gauss linear scheme unbounded and of second order is used. A PIMPLE algorithm is used for the pressure-velocity calculation procedure.

The CMC code is a finite volume solver, built as an extension of the reactingFoam solver. Full operator splitting is used for the terms in Eq. (10) in order to reduce the computational load. The conditional chemical source term is calculated using solver VODPK [Brown 1989]. The micromixing term is calculated with Tridiagonal Matrix Algorithm (TDMA) method. The first-order upwind and second-order central differencing schemes are used for conditional convection and subgrid conditional flux terms of Eq. (10). Time integration is performed by first-order Euler scheme. As mentioned, the $\mathrm{H}_{2} / \mathrm{O}_{2}$ reaction mechanism consists of 19 reversible elementary reactions between 9 species Li [2004]. The chemical source term in Eq. (10) were computed using the CHEMKIN ${ }^{\circledR}$ libraries.

The simulation time step is dynamically adjusted so that the Courant number never exceeds 0.35 . The execution time needed to simulate $3 \mathrm{~s}$ of combustion is approximately three weeks. The simulations are performed on an Intel Xeon E5-2680v2 10-core 2.7GHs cluster.

\section{BOUNDARY CONDITIONS}

As mentioned in the introduction, the principal objective is a detailed study on the boundary conditions. Therefore these are described in detail here.

The overview of the boundary conditions for the velocity, pressure, temperature and mixture fraction as implemented in OpenFOAM ${ }^{\circledR}$ [Christopher 2015], is provided in Table 1.

Table 1

Boundary Conditions implemented in OpenFOAM ${ }^{\circledR}$

\begin{tabular}{ccccc}
\hline & $\mathrm{U}[\mathrm{m} / \mathrm{s}]$ & $\mathrm{p}[\mathrm{Pa}]$ & $\mathrm{T}[\mathrm{K}]$ & $\mathrm{f}[-]$ \\
\hline Inlet & $\mathrm{WN} / \mathrm{RS}$ & zeroGradient & fixedValue=298 & fixedValue=1 \\
Co-flow & FixedValue=0.2 & zeroGradient & fixedValue=298 & fixedValue=0 \\
Sides & pressureInletOutletVelocity & totalPressure & inletOutlet & inletOutlet \\
Outlet & inletOutlet & zeroGradient & inletOutlet & inletOutlet \\
\hline
\end{tabular}


In LES, the inflow boundary conditions are important and the setting of the turbulent inflow conditions is not trivial. In this work, two different methods are used to generate the inflow boundary condition at the inlet. For the calculations named ' $\mathrm{WN}$ ' the standard OpenFOAM boundary condition -turbulentInlet, is used. The method on which this boundary is based consists of superimposing random fluctuations (White Noise - WN) generated by a standard random generator on the mean velocity profile. The velocity profile is assumed to follow the $1 / 7$ power law with $R e=10000$ (mean velocity equal to $34.8 \mathrm{~m} / \mathrm{s}$ ). The imposed fluctuations have an amplitude of $3 \%$ of the mean injection velocity.

For the calculations named 'RS' the method of Random Spots is used. Through this method at each time step, $\mathrm{M}$ spots are randomly placed in the space, with every $i^{\text {th }}$ spot having distribution of a component of the velocity fluctuation $f\left(x-x_{r i}^{(n)}\right)$, where $x_{r i}^{(n)}$ is the center of the $i^{\text {th }}$ spot [Kornev 2007]. The velocity fluctuation at the $n_{t h}$ time instant, $v_{n}^{\prime}$, is calculated as the sum of fluctuations produced by each spots:

$v_{n}^{\prime}(x)=\sum_{i=1}^{M} r_{i}^{n} f\left(x-r_{r i}^{n}\right)$

In Eq. (19) $r_{i} \in[-1,1]$ are random numbers and $x_{r i}$ is the random position of the spots.

In order to be able to use this boundary condition, several data should be provided:

- The inlet mean velocity profile. Here it is assumed to follow the 1/7 power law.

- The integral length scale which is the integral length between each spot at the inlet. Here it is chosen considering that in the self-similar region of a turbulent jet the lateral correlation is typically $0.3 r_{1 / 2}$, where $r_{1 / 2}$ is the radius at which the velocity is the half of the maximum [Pope 2000]. The value is set equal to $0.001 \mathrm{~m}$. Referring to the self-similar region, lateral correlation is slightly lower close to the centerline. Note that in any case the length scale cannot be smaller than the smallest cell size (in this case equal to $0.0008 \mathrm{~m}$ ).

- The Reynold stresses. These are given at each spot through a non-uniform list of symmetric tensors. At this purpose for each component the radial profile in the self-similar region is used [Pope 2000].

It is noted that neither of the two methods generate turbulence structures that are correlated in time (i.e., there is no notion of turbulence integral time scale). Improving this is beyond the scope of the present study.

However, also the boundary conditions at the sides of the computational domain deserve further attention. First of all, a mixed boundary condition is assigned for velocity at sides of the domain, setting the velocity equal to zero for any outward flow and calculating the inlet velocity from the pressure field. Secondly, a Dirichlet boundary condition, named totalPressure, is assigned for pressure at the sides: with this boundary condition pressure is adjusted according to the velocity changes. As reported in the OpenFOAM ${ }^{\circledR}$ user guide [Cristopher 2015] this combination of boundaries conditions are suited for patches where some inflow could occur, which is the case of boundaries surrounding a jet through a nozzle.

\section{RESULTS}

Figure 4 gives an overview of the contours of time averaged velocity and temperature fields for the Random Spot (RS) and White Noise (WN) calculations. Time averaging starts after $0.5 \mathrm{~s}$ and statistics are collected over a period of $2.5 \mathrm{~s}$. The iso-lines of the stoichiometric mixture fraction $f=f_{s t}$ and the position of the measured radial profiles are indicated in the slices as well. The contours in Figure 4 show that when White Noise is used the jet break-up occurs later, leading to a longer flame. In this case, the inlet 'turbulence' dies due to lack of any temporal and special coherence. 

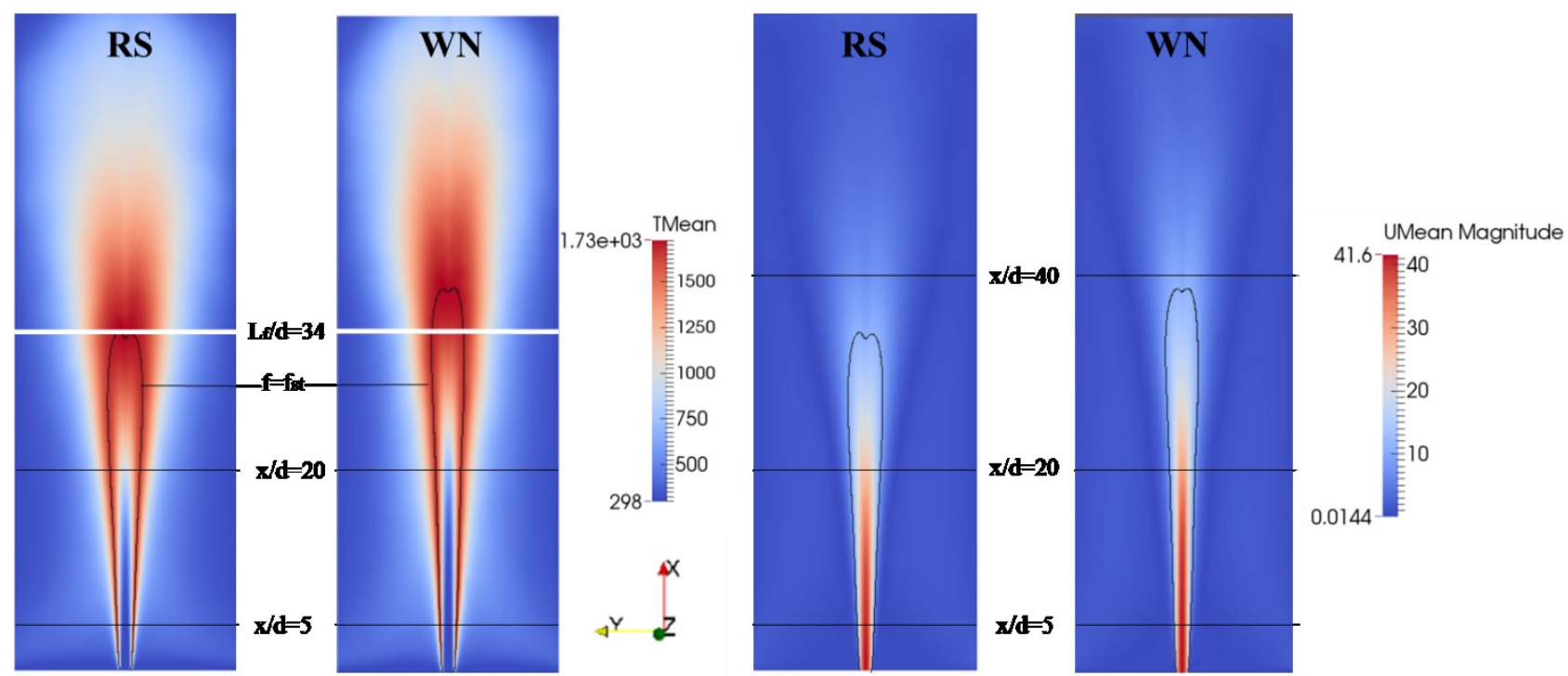

Figure 4. Contour plots of time-averaged temperature (left) and velocity (right) fields as obtained with the Random Spots (RS) and White Noise (WN) inlet boundary conditions.

The radial profiles of mean and rms velocity at $\mathrm{x} / \mathrm{d}=5,20,40$ are shown in Figure 5 and Figure 6. Clear differences are observed between the WN and RS results.

$$
\mathbf{x} / \mathbf{d}=\mathbf{5}
$$
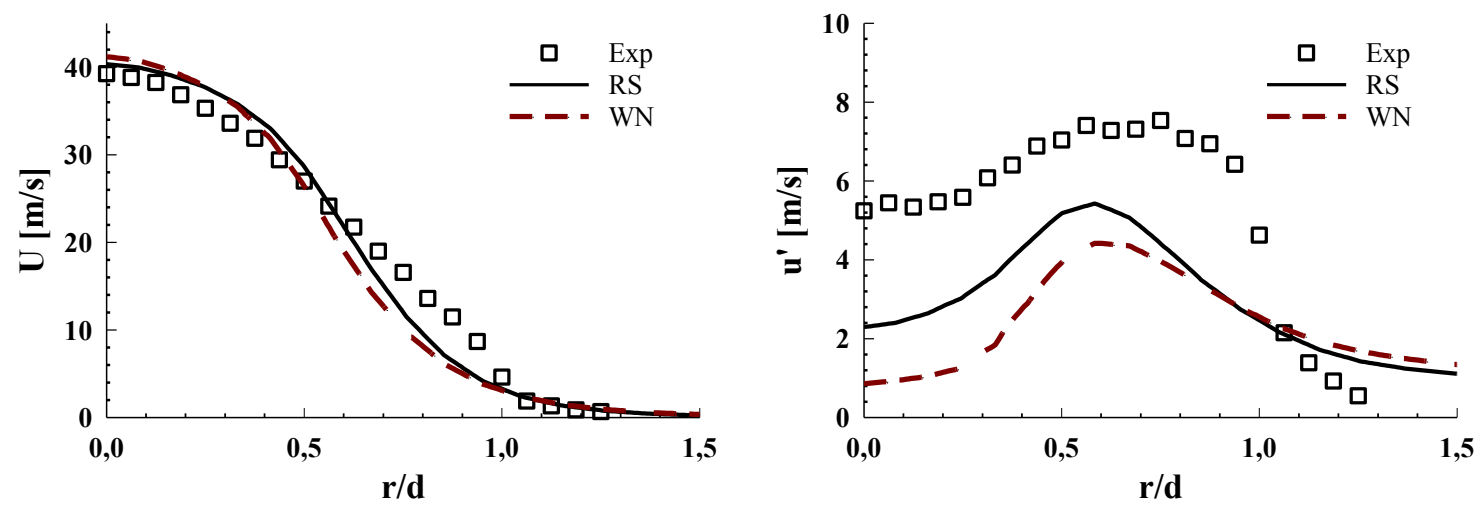

$\mathbf{x} / \mathbf{d}=\mathbf{2 0}$
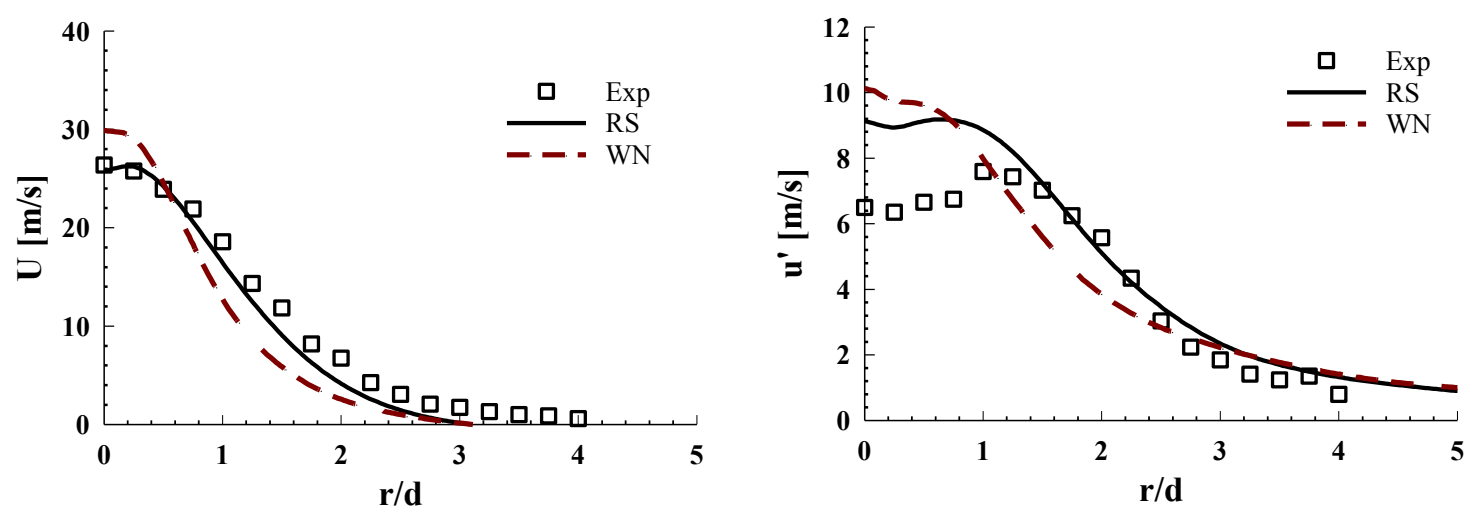

Figure 5. Favre-averaged mean (left side) and rms velocity (right side) radial profiles at locations $\mathrm{x} / \mathrm{d}=5, \mathrm{x} / \mathrm{d}=20$. 

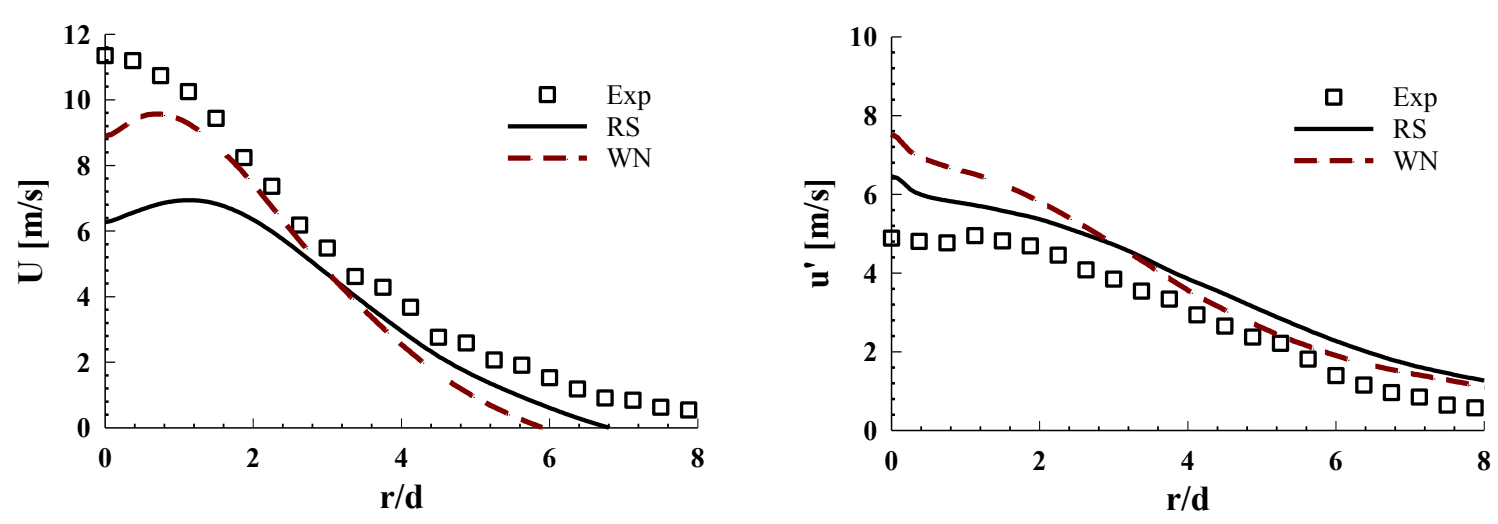

Figure 6. Favre-averaged mean (left side) and rms velocity (right side) radial profiles at location $\mathrm{x} / \mathrm{d}=40$.

At $\mathrm{x} / \mathrm{d}=5$ the maximum $\mathrm{rms}$ velocity is observed at $\mathrm{r} / \mathrm{d}=0.6$ for both calculations, corresponding to the radial location where the radial gradient in velocity is the highest. The level of velocity fluctuations is very low with WN, as mentioned above (the inlet turbulence dies). Also the velocity fluctuations obtained with the RS method are lower than what is reported in the experiments. This may partly be due to the absence of temporal correlations between the random spots, as mentioned. Indeed, in Forkel [2011], it is shown that when inlet profiles generated by separate LES pipe flow simulations are imposed, good agreement is observed with experiments. In this case, turbulent structures are correlated in time and space. Overall the RS results are in better agreement with the experimental data, despite a significant under-prediction of the mean velocity on the centerline at $\mathrm{x} / \mathrm{d}=40$. The seemingly better result with $\mathrm{WN}$ is misleading, as can be understood from Figure 9 (see below). Note also that the maximum velocity is not on the centerline at $\mathrm{x} / \mathrm{d}=40$ in both simulations, in contrast to the experimental data. Figure 7 shows the mean and rms velocity profiles along the centerline. Close to the nozzle exit $(\mathrm{x} / \mathrm{d}<10)$ the velocity fluctuations drop to almost zero for the WN calculations, while they remain around $3 \mathrm{~m} / \mathrm{s}$ for the RS simulations. This confirms once again that turbulence dies in the WN calculations. As a consequence, the mean velocity does not decay sufficiently quickly. In contrast, the mean velocity starts to decrease immediately due to the turbulence, in much better agreement with the experiments. In the far field region $(x / d>20)$ a significant under-prediction is observed, though. With WN the velocity fluctuations revive from $\mathrm{x} / \mathrm{d}>10$ onwards, but then the fluctuations become excessive, far from the experimental values. Through the WN method, in fact, the perturbations introduced at the inlet are able to induce only intensity without any relations between turbulence scales. Also the RS velocity fluctuations increase at $\mathrm{x} / \mathrm{d}>10$, up to higher values than measured in the experiments. Nevertheless, the over-all level is in much better agreement with the experimental data.
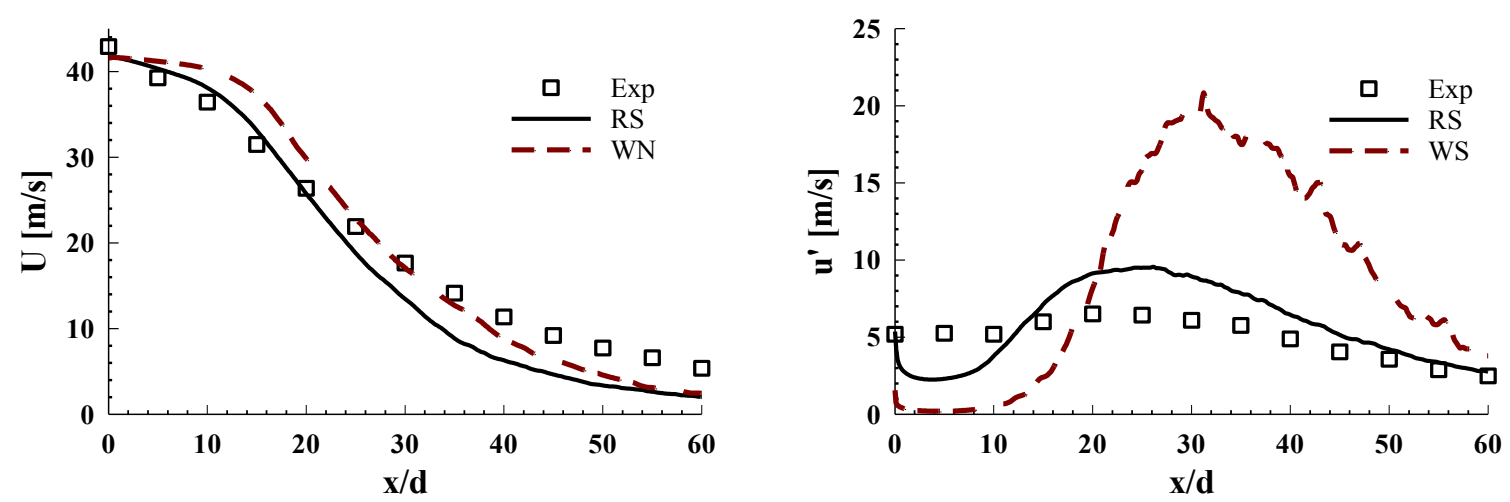

Figure 7. Favre-averaged mean (left side) and root mean square (right side) velocity profiles at the centerline. 

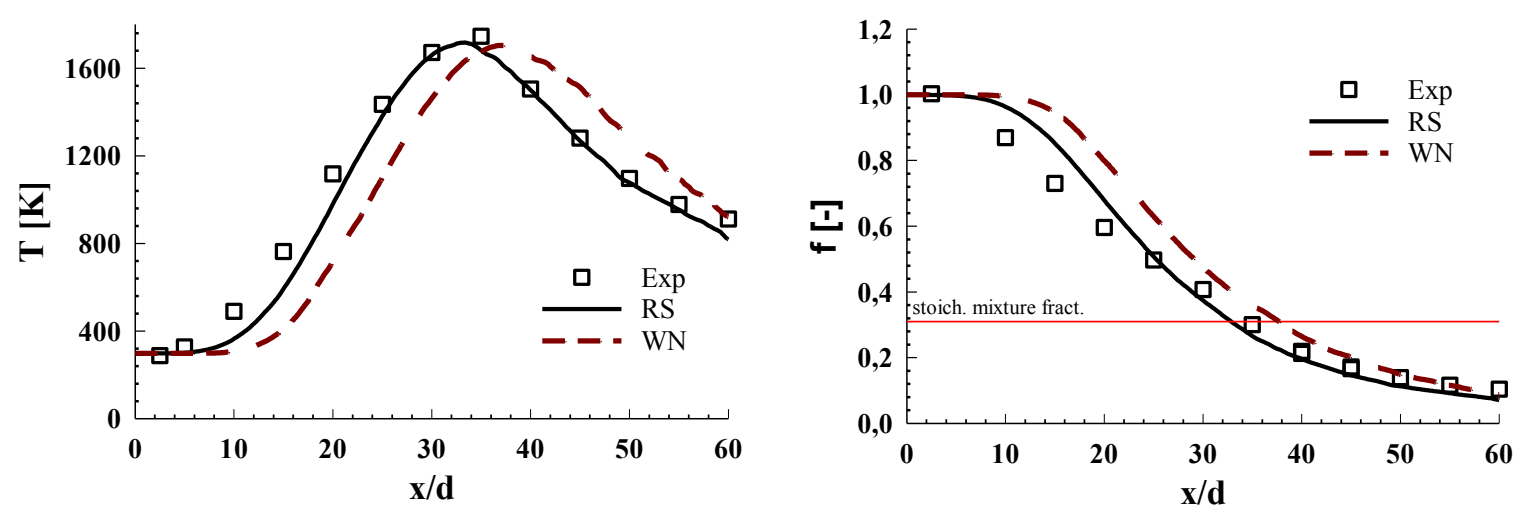

Figure 8. Favre averaged mean temperature (left side) and mixture fraction (right side) at the centerline.

For obvious reasons, the flow field has a direct impact on the evolution of mean temperature and mixture fraction, f, shown at the centerline in Figure 8 and at two downstream locations in Figure 9.

\section{$\mathbf{x} / \mathbf{d}=\mathbf{5}$}
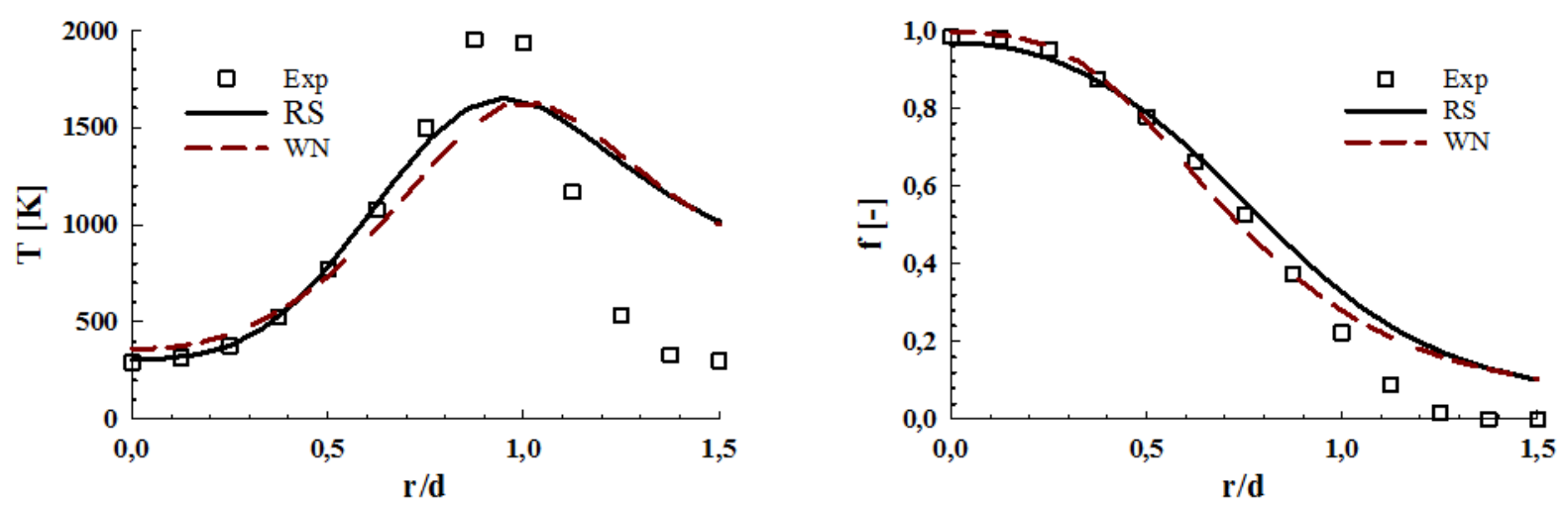

$\mathbf{x} / \mathbf{d}=\mathbf{2 0}$
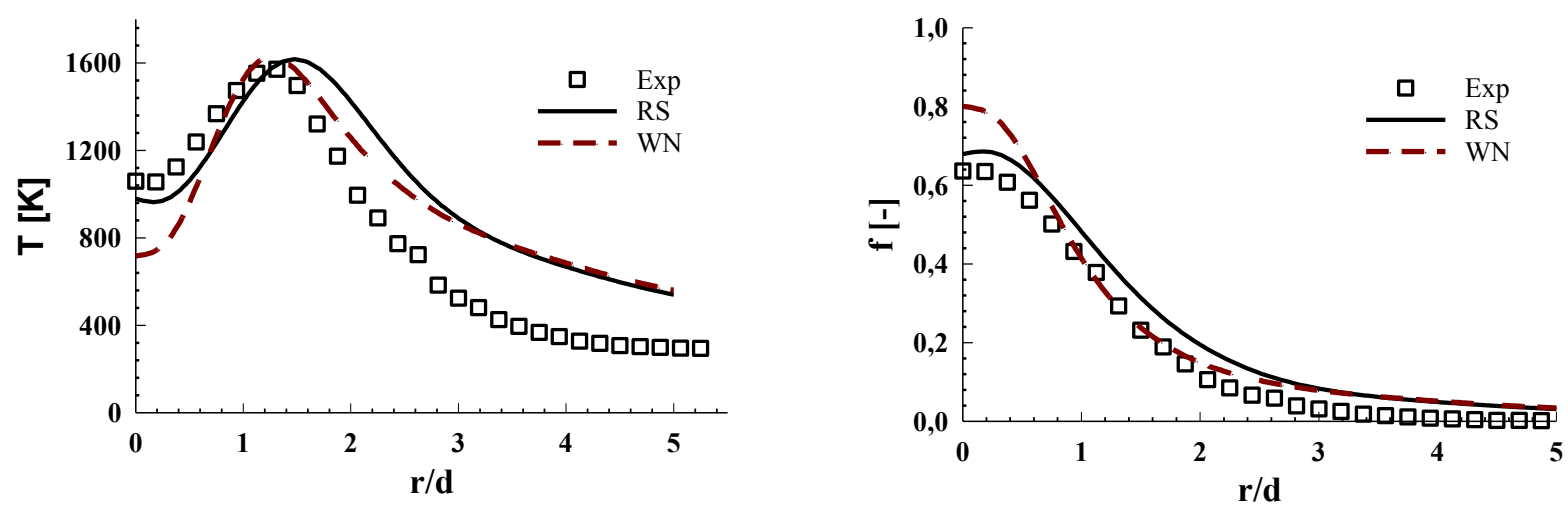

Figure 9. Radial profiles of Favre averaged mean temperature (left side) and mixture fraction (right side) at location $\mathrm{x} / \mathrm{d}=5$ and $\mathrm{x} / \mathrm{d}=20$.

Figure 8 shows that the maximum temperature is reached in the experiments at location $\mathrm{x} / \mathrm{d}=34$, where the stoichiometric mixture fraction $f_{s t}=0.31$ is obtained. In the RS case the maximum is shifted slightly upstream, with deviation between the experimental and simulation location value as low as $2 \%$. In the $\mathrm{WN}$ case the maximum temperature is shifted downstream (with an error of about $10 \%$ ) and simulation results do not match the experimental data well throughout the entire profile. The evolutions of mean temperature and mean mixture fraction obtained in the RS simulations clearly follow the experimental results very closely. An error analysis of the absolute value and the position 
of the maximum flame temperature, together with their values at the centerline, is presented in Table 2.

Table 2. Error analysis on the position of the maximum flame temperature

\begin{tabular}{cccccc} 
& x/d Exp. & x/d RS & r/d WN & Error (\%) RS & Error (\%) WN \\
\hline Centerline & 34.0 & 33.2 & 37.6 & 2.30 & 10.7 \\
& $T_{\max }$ Exp. & $T_{\max } \mathrm{RS}$ & $T_{\max } \mathrm{WN}$ & Error (\%) RS & Error (\%) WN \\
\hline Centerline & 1745.7 & 1716.9 & 1704.9 & 1.65 & 2.30
\end{tabular}

At $\mathrm{x} / \mathrm{d}=5$ the experimental maximum temperature is $T_{\max }=1950 \mathrm{~K}$ and, due to differential diffusion effect, this is not reached in either of the calculations. This was also observed in LES simulation of Forkel [2000] while in Maragkos [2014], who included differential diffusion, the maximum temperature at this location was well predicted.

Figure 9 confirms that the RS results are also in much better agreement with the experimental data when radial profiles are examined. In particular at $\mathrm{x} / \mathrm{d}=20$, the profiles are in much closer agreement. It is important to note that the results are also significantly affected by the boundary conditions at the sides. This is discussed below (Figure 13).

The Favre mean temperature and $\mathrm{H}_{2} \mathrm{O}$ mass fraction plotted as a function of mixture fraction at $\mathrm{x} / \mathrm{d}=5$ are shown in Figure 10 and Figure 11 respectively. As also visible in above figures, the temperature is under-predicted at the lean side of the flame, compared to experimental data. This is due to differential diffusion effects, which lead to higher temperatures [Maragkos 2014]. Further downstream (e.g., at $\mathrm{x} / \mathrm{d}=20$ ), where differential diffusion effects are less important, there is perfect agreement between experimental and simulation data as reported. At $\mathrm{x} / \mathrm{d}=20$ higher mixture fraction values for the WN case can be observed and wider flame is present what can be clearly seen in Figure 10 and Figure 11. There are points were the gaseous mixture is richer than expected which confirms that mixing, hence turbulence, is not well represented.
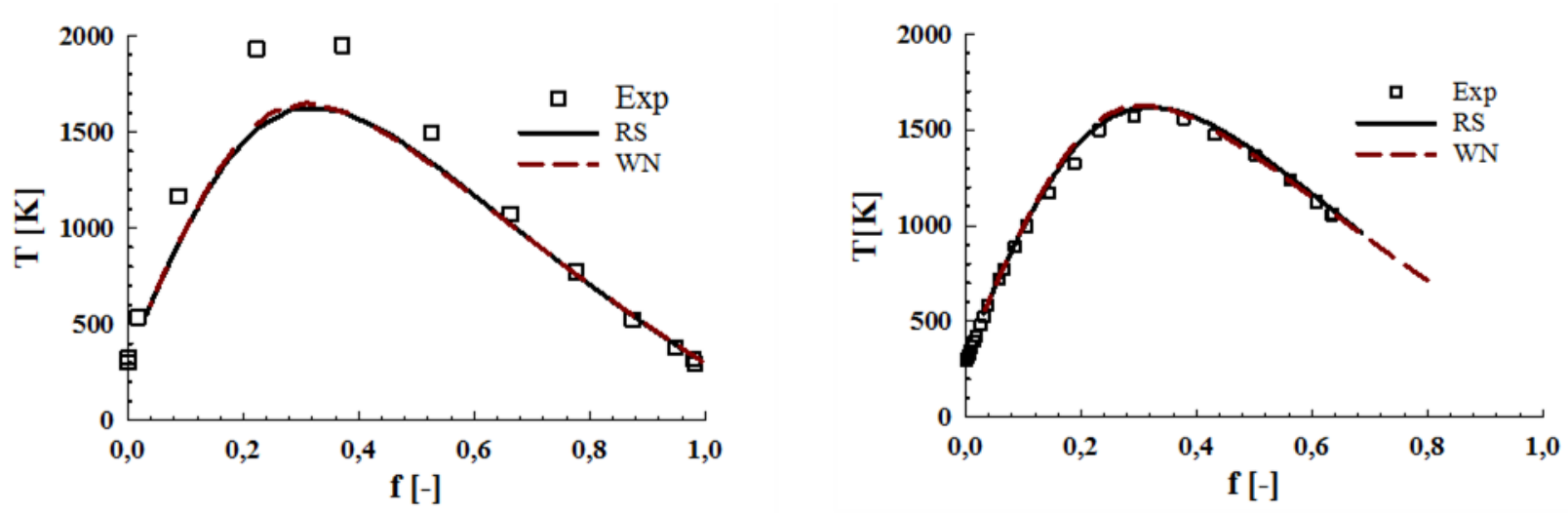

Figure 10. Unconditional profiles for temperature as function of mixture fraction at location $\mathrm{x} / \mathrm{d}=5$ (left) and $\mathrm{x} / \mathrm{d}=20$ (right). 

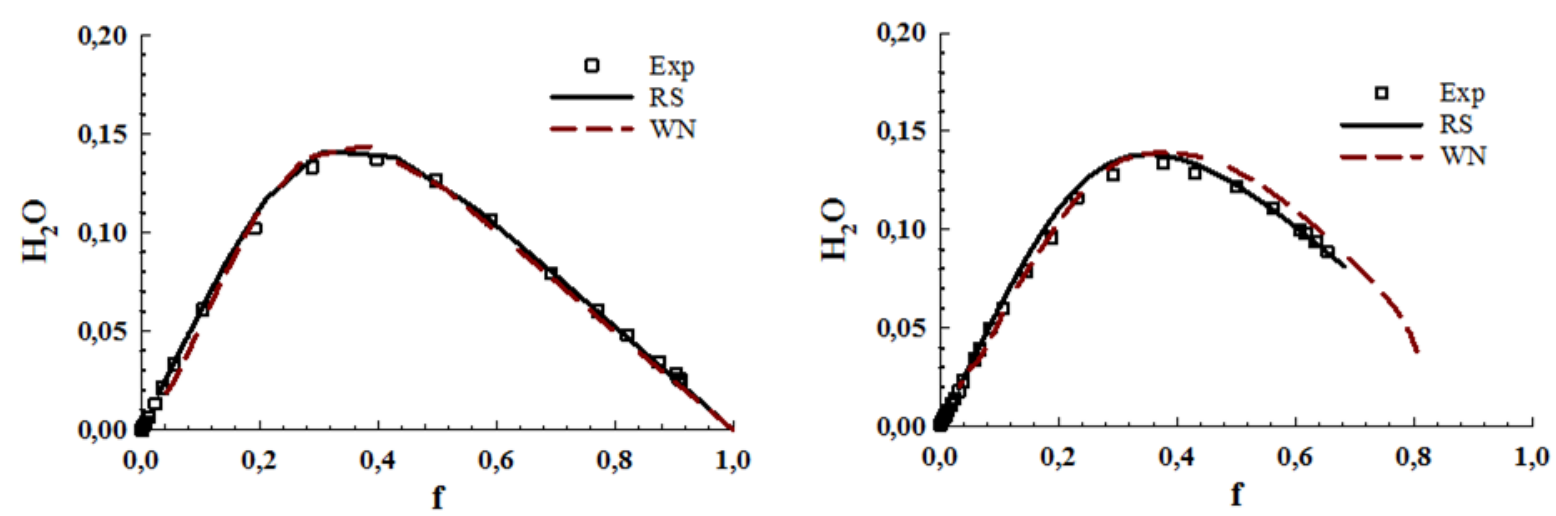

Figure 11. Unconditional profiles for temperature and $\mathrm{H}_{2} \mathrm{O}$ mass fraction as function of mixture fraction at location $\mathrm{x} / \mathrm{d}=5$ (left) and $\mathrm{x} / \mathrm{d}=20$ (right).

Focusing on the radial profiles at $\mathrm{x} / \mathrm{d}=5$ and $\mathrm{x} / \mathrm{d}=20$ (Figure 9), the over-prediction of temperature and mixture fraction at the lean side of the flame is striking. The reason for this has been identified to be the boundary conditions at the sides. In order to illustrate this, the RS simulation results are compared to results, labeled RS*, where the open side boundaries are replaced by a solid boundary (no-slip velocity boundary condition at the sides of the domain). Figure 12 shows the impact on the mean mixture fraction field. This is directly related to unrealistic radially outward flow close to the nozzle with the original boundary condition, forcing fuel into a region where only oxygen is expected. In combination with the combustion model this causes chemical reaction and a temperature rise at the sides of the actual flame (see also Figure 4: the colour is light blue in that region). The outflow at the sides is shown in Figure 14 for RS case and it is clearly absent when new set of boundary conditions is used ( $\left.\mathrm{RS}^{*}\right)$.

Results for the RS* case are reported only in the lower half of the domain $(\mathrm{x} / \mathrm{d}<30)$ and cannot be discussed for the locations further downstream as even with the new boundary conditions, the flame interacts with the walls higher up and numerical instabilities are present. Nevertheless, using the RS* case in the region close to the nozzle leads to a better understanding of the actual behavior of the flame in that region when the artificial outflow at the sides is excluded.

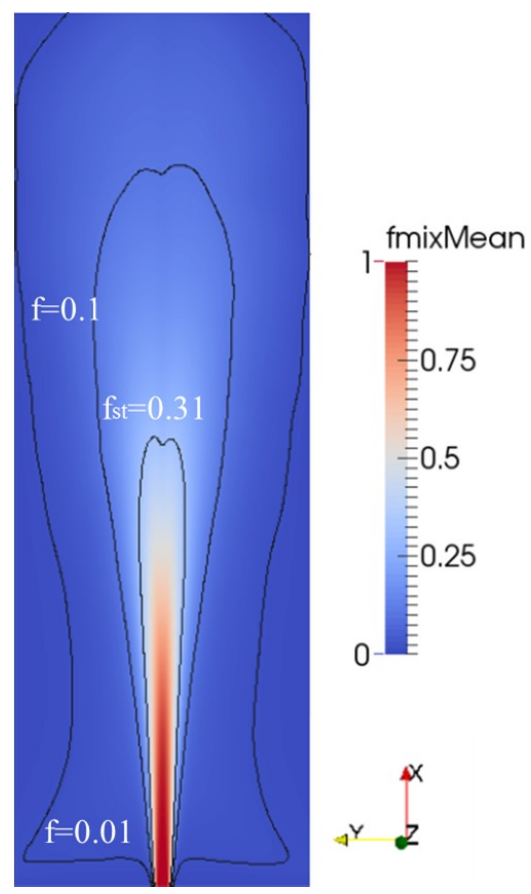

Figure 12. 2D contour plot of mixture fraction and mixture fraction iso-lines for RS case. 


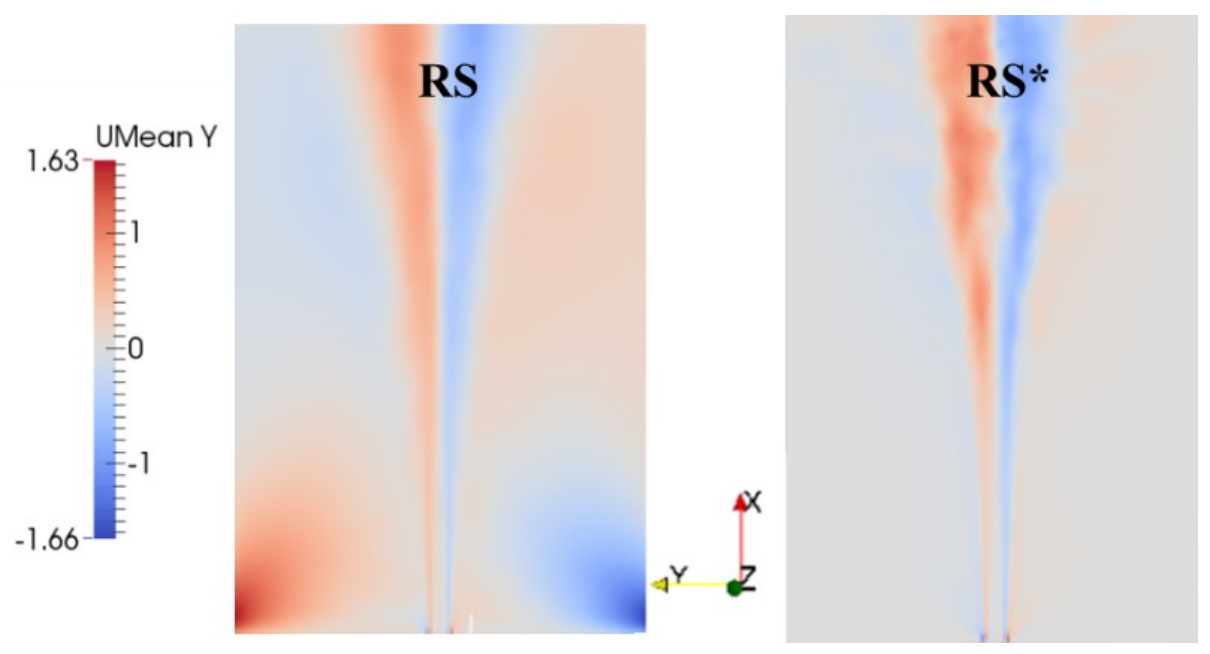

Figure 13. 2D contour plot of mean velocity (y component) at lower half $(\mathrm{x} / \mathrm{d}<30)$ domain.

Left: RS case; right: RS* case.

$\mathbf{x} / \mathbf{d}=\mathbf{5}$
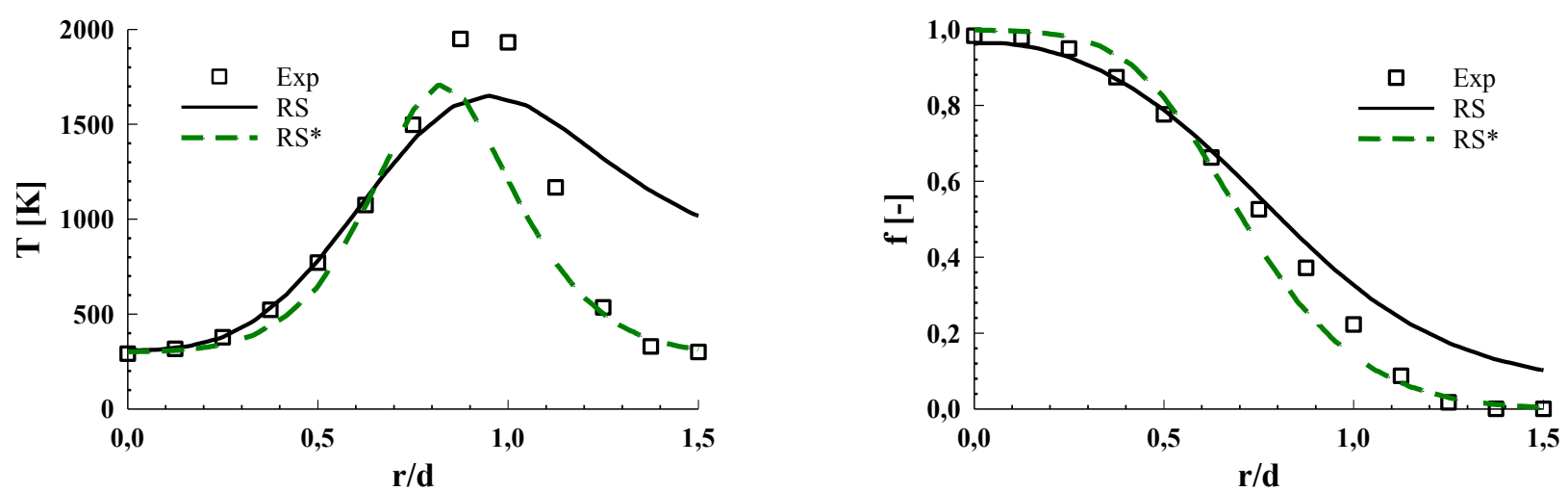

$\mathbf{x} / \mathbf{d}=\mathbf{2 0}$
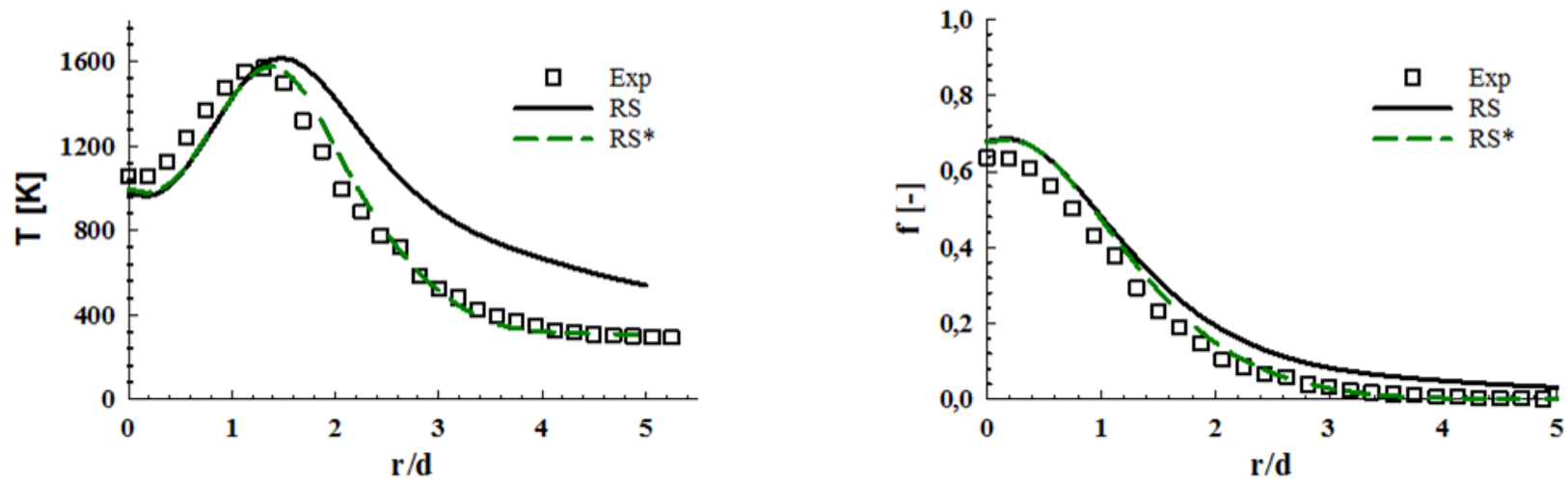

Figure 14. Favre averaged mean temperature (left side) and mixture fraction (right side) at locations $\mathrm{x} / \mathrm{d}=5$ and $\mathrm{x} / \mathrm{d}=20$.

Figure 14 confirms the very beneficial impact on the results in physical space. The RS* results are in excellent agreement with the experimental data at $\mathrm{x} / \mathrm{d}=20$ and also in much better agreement with experimental data than the RS results at $\mathrm{x} / \mathrm{d}=5$. This clearly illustrates that the impact of the side boundary conditions must not be under-estimated for this case. 
Profiles of species mass fractions at location $\mathrm{x} / \mathrm{d}=5$ are presented in Figure 15. There is a good agreement between experimental and numerical results but still, as discussed above for temperature, the effect of the outflow at sides influences all the radial profiles at the lean side region.

a)

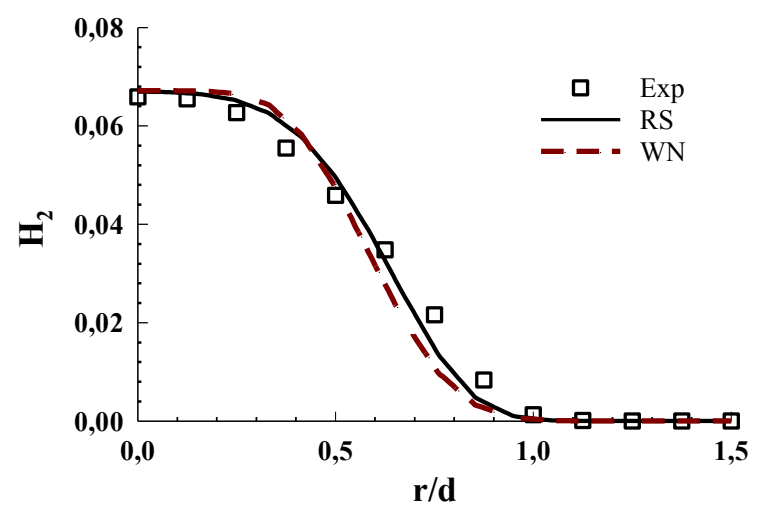

c)

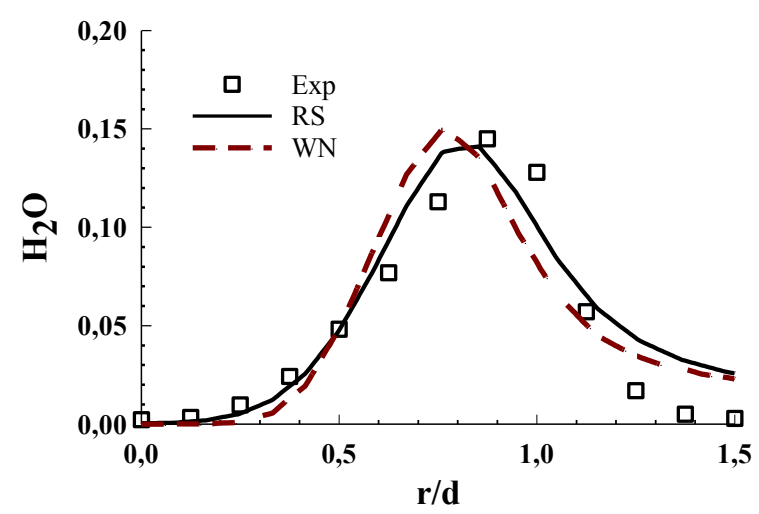

b)

$$
\mathbf{x} / \mathbf{d}=\mathbf{5}
$$

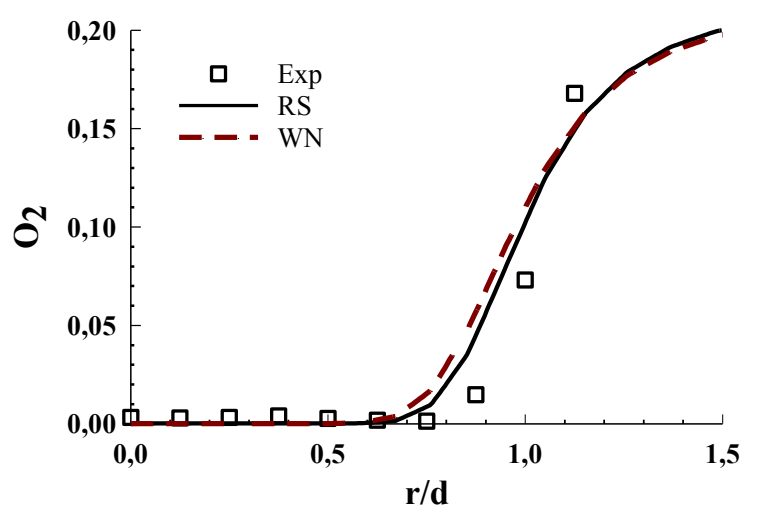

d)

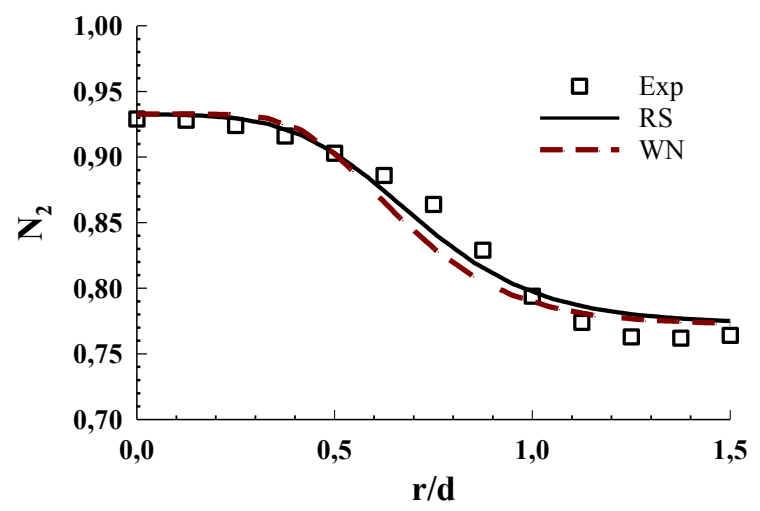

Figure 15. Favre averaged species mass fraction at location $\mathrm{x} / \mathrm{d}=5$ for (a) $\mathrm{H} 2$, (b) $\mathrm{O} 2$, (c) $\mathrm{H} 2 \mathrm{O}$, (d) N2.

\section{CONCLUSIONS}

CFD simulations, combining the LES/CMC approaches, have been presented for a turbulent nonpremixed flame, with a mixture of hydrogen and nitrogen as fuel. The LES equations were solved using OpenFOAM, an in-house finite-volume code was used for the CMC equations.

In order to illustrate the effect of turbulence, two types of inlet boundary conditions have been applied: using a fixed mean inlet velocity profile, turbulence was superposed either as Random Noise (i.e., complete absence of temporal and spatial correlations in the imposed velocity fluctuations) and a method of Random Spots (where there is spatial correlations through the imposed length scale, but no temporal correlation in the imposed velocity fluctuations). As expected, the impact on the results is significant and the method of Random Spots is in much better agreement with the experimental data in terms of Favre-averaged profiles of velocity, mixture fraction, temperature and species. This has been explained by the fact that the synthetic turbulence, imposed at the inlet, does not die completely. Further improvement, introducing also temporal correlations in the fluctuations in the Random Spots method, is possible, though.

An important second observation is that also the boundary conditions applied at the sides of the computational domain can have a strong impact on the results. Artificial sideward outflow close to 
the inlet leads to unrealistic mixing of fuel and oxygen and, consequently, to unrealistically high temperatures in that part of the domain. This issue has been resolved by replacing the side boundary conditions, originally implemented as open (with possible inflow and outflow), by walls. This part of the study has been limited to the region sufficiently close to the nozzle $(\mathrm{x} / \mathrm{d}<20)$, because the flame still interacts with the walls higher up in the domain.

\section{Acknowledgement}

This research has been funded by Ghent University (Belgium) through GOA project BOF16/GOA/004 (PREditction of Turbulent REactive Flows - http://www.pretref.ugent.be/) The authors strongly acknowledge Prof. Mastorakos (The University of Cambridge) and dr. Garmory (Loughborough University) for providing the in-house CMC code used in this study.

\section{REFERENCES}

Boussinesq, J., 1877. La theorie des eaux courantes, Paris: Imprimerie Nationale.

Brown, P. N. \& Hinmarsh, A. C., 1989. Reduced storage matrix methods in stiff ode systems. Journal of Computational Applied Mathematics, Volume 31, pp. 40-91.

Cristopher , J., 2015. OpenFOAM User Guide. [Online]

Available at: http://www.openfoam.org/docs/OpenFOAM Documentation

Forkel, H. \& Janicka, J., 2000. Large Eddy simulation of a turbulent hydrogen diffusion flame. Flow, Turbulence and Combustion, 65(2), pp. 163-175.

Fureby, C. \& Tabor, G., 1997. Mathematical and physical constraints on large-eddy simulations. Theoretical Computational Fluid Dynamics, Volume 9, pp. 85-102.

Garmory, A. \& Mastorako, E., 2013. Sensitivity analysis of LES-CMC predictions of piloted jet flames. International Journal of Heat and Fluid Flow, Volume 39, pp. 53-63.

Garmory, A. \& Mastorakos, E., 2011. Capturing localised extinction in Sandia Flame F with LES-CMC. Proceedings of the Combustion Insitute, Volume 33, pp. 1673-1680.

Garmory, A. \& Mastorakos, E., 2015. Numerical simulation of oxy-fuel jet flames using unstructured LESCMC. Proceedings of the Combustion Institute, Volume 35, pp. 1207-1214.

Germano, M., Piomelli, U., Moin, P. \& Cabot, W. H., 1991. A dynamic subgrid-scale eddy viscosity model. Physics Fluids A, Volume 3, pp. 1760-1765.

Klimenko, A. Y. \& Bilger, R. W., 1999. Conditional moment closure for turbulent combustion. Prgress in Energy and Combustion Science, Volume 25, pp. 595-687.

Kornev, N. \& Hassel, E., 2007. Method of random spots for generation of synthetic inhomogeneous turbulent fields with prescribed autocorrelation functions. Communications in Numerical Methods in Engineering, Volume 23, pp. 35-43.

Li, J., Zhao, Z., Kazakov, A. \& Dryer, F., 2004. An Updated Comprehemsive Kinetic Model of Hydrogen Combustion. International Journal of Chemical Kinetics, Volume 36, pp. 566-575.

Magnussen, B. F., 2005. The Eddy Dissipation Concept a bridge between science and technology. Lisbon, ECCOMAS Thematic Conference on Computational Combustion.

Magnussen, B. F. \& Erstevag, I. S., 2000. The Eddy Dissipation turbulence energy cascade model,. Combustion Science Technology, Volume 159, pp. 213-236. 
Maragkos, G., Rauwoens, P. \& Merci, B., 2014. Assessment of a methodology to include differential diffusion in numerical simulations of a turbulent flame. International journal of Hydrogen Energy, Volume 40, pp. $1212-1228$.

Meier, W., Prucker, S., Chao, M. H. \& Stricker, W., 1996. Characterization of a turbulent H2/N2/air jet diffusion flames by single-pulse spontaneous raman scattering.. Combust. Sci. Technol, Volume 63, pp. 293312.

Mueller, M. A., Kim, T. J., Yetter, R. A. \& Dryer, F. L., 1999. Flow reactor studies and kinetic modeling of the H2/O2 reaction. International journal of Chemical Kinetics, Volume 31, pp. 113-125.

O'Brien, E. E. \& Jiang, T. L., 1991. The conditional dissipation rate of an initial binary scalar in homogeneous turbulence. Phys. Fluids, Volume 3, pp. 3121-2123.

Panjwani, B., Erstevag, A., Gruber, A. \& Rian, K. E., 2010. Turbulence combustion closure model based on the Eddy dissipation concept for large eddy simulation. Advances in Fluid Mechanics VIII, Volume 69, pp. 27-38.

Pera, C., Reveillon, J., Vervisch, L. \& Domingo, P., 2006. Modeling subgrid scale mixture fraction variance in LES of evaporating spray. Combustion Flame, Volume 146, pp. 635-648.

Pfuderer, D. G. et al., 1996. Turbulence Modulation in Jet Diffusion Flames: Modeling and Experiments. Combustion and Flame, Volume 106, pp. 302-317.

Pitsch, H., Chen, M. \& Peters, N., 1998. Unsteady Flamelet Modeling of Turbulent Hydrogen-Air Diffusion Flames. Twenty-Seventh Symposium (International) on Combustion, pp. 1057-1064.

Poinsot, T. \& Veynante, T., 2001. Theoretical and Numerical Combustion. Institut de Mecanique des Fluides Toulose: Edwards.

Pope, S. B., 2000. Turbulent flows. 1 ed. Cambridge: University of Cambridge.

Schumann, U., 1975. Subgrid scale model for finite differences simulation of turbulent flows in plane channels and anuli. Journal Computational Physics, Volume 18, pp. 376-40.

Stankovic, I., Mastorakos, E. \& Merci, B., 2013. LES-CMC simulations of different auto-ignition regimes of hydrogen in a hot turbulent air co-flow. Flow Turbulence and Combustion, Volume 90, pp. 583-604.

Zhang, H. \& Mastorakos, E., 2016. Prediction of Global Extinction Conditions and Dynamics in Swirling Nonpremixed Flames Using LES-CMC. Flow turbulence Combustion, Volume 96, pp. 864-889. 